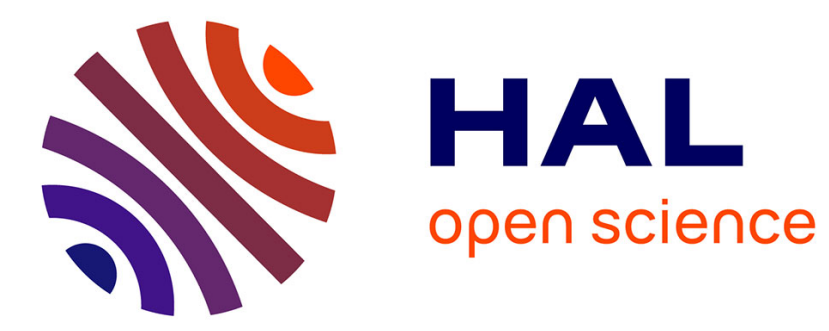

\title{
De la reconnaissance à l'empathie : éléments pour une anthropologie des dimensions socio-culturelles de la souffrance à partir des récits des réfugiés syriens en exil au Liban
}

Jean-Baptiste Pesquet

\section{To cite this version:}

Jean-Baptiste Pesquet. De la reconnaissance à l'empathie: éléments pour une anthropologie des dimensions socio-culturelles de la souffrance à partir des récits des réfugiés syriens en exil au Liban. CORPS : Revue Interdisciplinaire, 2021, 78, pp.79-89. halshs-03120636

\section{HAL Id: halshs-03120636 \\ https://shs.hal.science/halshs-03120636}

Submitted on 25 Jan 2021

HAL is a multi-disciplinary open access archive for the deposit and dissemination of scientific research documents, whether they are published or not. The documents may come from teaching and research institutions in France or abroad, or from public or private research centers.
L'archive ouverte pluridisciplinaire HAL, est destinée au dépôt et à la diffusion de documents scientifiques de niveau recherche, publiés ou non, émanant des établissements d'enseignement et de recherche français ou étrangers, des laboratoires publics ou privés. 


\section{DE LA RECONNAISSANCE À L'EMPATHIE : ÉLÉMENTS POUR UNE ANTHROPOLOGIE DES DIMENSIONS SOCIO-CULTURELLES DE LA SOUFFRANCE A PARTIR DES RÉCITS DES RÉFUGIÉS SYRIENS EN EXIL AU LIBAN}

Jean-Baptiste PESQUET

La guerre civile en Syrie produit des expériences de violences extrêmes que l'ethnographie auprès des réfugiés en exil permet de recueillir sous la forme de récits. Cet article analyse les relations entre pouvoir, savoir et éthique dans l'expression des souffrances des réfugiés syriens au Liban à partir d'une recherche réalisée entre 2012 et $2016^{1}$. Il montre que les professionnels de santé mentale reformulent les récits des réfugiés pour les inscrire dans une tradition discursive moderne et séculière de la souffrance. Dans cette conception, les pratiques religieuses ont rarement une valeur thérapeutique alors qu'elles sont essentielles pour les réfugiés dans la gestion quotidienne des souffrances de guerre. Largement fondée sur la suspicion d'une instrumentalisation pour obtenir des aides, l'approche des professionnels objectivise les souffrances pour soigner, ce qui peut paradoxalement provoquer des blessures morales. Dès lors, une approche scientifique soucieuse d'éviter de nouvelles violences peut s'appuyer d'une part sur les théories de la reconnaissance et d'autre part sur la notion d'empathie en phénoménologie pour proposer une lecture critique de ces discours.

\section{SITUATION ET DÉFINITION DES RÉCITS DE SOUFFRANCE}

Le corpus de propos que je rassemble sous la catégorie "récits de souffrance » forme un type de récits au sein de l'ensemble des témoignages recueillis auprès des réfugiés syriens au Liban sur leur exil. Les «récits politiques» proposent des analyses de la guerre en fonction de différents positionnements politiques. Les « récits d'exil» décrivent les raisons du départ de Syrie et les conditions de vie au Liban sous le registre des vulnérabilités. Les " récits de souffrance » quant à eux sont caractérisés par la présence de trois thématiques: l'humanité (insani, insanieh), les animaux (haywanat) et Dieu (Allah). Ils peuvent prendre la forme de lamentations lorsqu'ils dénoncent une inégalité de traitement (ex : "Où est l'humanité ? Nous sommes moins bien traités que des animaux ! »), ou prendre la forme d'insultes pour déshumaniser l'ennemi (ex : « Bachar alAssad n'est pas un lion, c'est un chien. Il est sale »). Dieu occupe la position suprême dans la taxinomie qui se dégage du registre des limites de l'humanité. Il est pris pour témoin des souffrances auxquelles les institutions humaines, comme les organisations humanitaires ou les États, ne répondent pas (ex: "L'humain a-t-il si peu de valeur à vos yeux ? Comment cela est-il possible ? C'est interdit, Dieu n'accepte pas ceci. »). L'enjeu langagier du registre de l'inhumanité est de savoir si les spectateurs de la souffrance partagent encore une même " nature humaine » avec les réfugiés dont la forme de vie a été brisée par la violence (Das, 2016).

L'aide humanitaire apportée par les différentes organisations prend en charge la douleur dans ses aspects physiques et psychologiques en s'appuyant sur des savoirs tels que la médecine, la psychologie clinique ou la psychiatrie. En face de ces acteurs, les réfugiés n'expriment pas leurs souffrances en termes de "santé mentale » (al saha al 'aqliah). Ils recourent à des expressions autres, telles que «je suis fatigué » (ana ta'ban) ou "fatigué psychologiquement» (nafsiti ta'banah). Les morts sont considérés comme des martyrs (chouhada), un terme qui recouvre tous les individus morts de manière violente dans le contexte de guerre. Il inclut les hommes morts au combat, les victimes de bombardements, de tortures, de maladie ou d'épuisement lors d'un siège. À ce vocabulaire, s'ajoutent des pratiques religieuses d'apaisement, telles que les prières individuelles et des cérémonies collectives. Ces savoirs différenciés de compréhension, d'expression et de gestion de la souffrance se rencontrent dans les entretiens entre professionnels de santé et réfugiés. 
L'analyse de ces échanges révèle alors des relations de pouvoir qui s'expriment notamment au travers des reformulations de ces récits.

\section{REFORMULATION DES RÉCITS ET PRODUCTION D'UN OBJET-SOUFFRANCE}

Pour illustrer ce point nous proposons l'analyse d'un entretien avec Jasmine, psychologue pour une association libanaise de protection de l'enfance. Lors d'un de mes différents sur son lieu de travail, Jasmine m'explique avoir suivi en consultation une enfant syrienne de onze ans, qui a été violée par un épicier en Syrie. Nous convenons de prendre un temps dans un bureau pour qu'elle me présente son analyse de la situation lors d'un entretien plus formel ${ }^{2}$. Jasmine me rapporte ainsi le récit de l'enfant, qui après avec acheté des légumes, est interpellée dans la rue par un homme. Elle ne lui répond pas et s'éloigne : «Et là elle [l'enfant syrienne] a dit qu'il a pu l'attraper et la ramener alors qu'elle était loin de lui. Il y avait quelque chose de... presque "cartoonish" [fantastique] dans la main qui s'allonge. Parce qu'elle a dit : "Il n'a pas bougé, il n'a pas couru, il m'a juste tirée vers lui". Bien sûr, elle est restée très discrète dans les mots qu'elle utilisait, elle a dit : "Il m'a obligée à faire quelque chose de pas bien" [chi mish mniha], quelque chose de "honteux" ['ayeb]. Elle a dit aussi que, tout d'un coup, tout le monde avait disparu de la rue et qu'elle n'avait plus de voix. Elle ne comprenait pas ce qui s'était passé. Elle avait décrit la personne à l'assistante sociale comme un monstre [wahesh], mais elle a aussi dit que c'était un homme. »

L'enfant ne raconte son viol qu'à sa mère, puis entre dans une période de mutisme. Ses parents font alors appel à un religieux (cheikh) qui accuse un djinn et propose de prier avec elle. Alors que ce dernier lui a dit de fermer les yeux et prie, l'enfant voit apparaître une dame blanche qui lui annonce qu'elle a tué le monstre. Mais Jasmine ne croit pas aux djinns, et elle apprend après quelques séances que ni la fille ni la mère ne croient dans l'intervention d'un djinn. S'ajoute ensuite un second élément qui vient confirmer son interprétation : «Au Liban, elle est partie chez le couturier, un vieil homme. C'est un peu le même contexte. Elle y va seule. Il lui demande de montrer sa culotte, un truc comme ça. Elle lui dit : "bah non pourquoi je te montrerais ma culotte ?" et lui dit : "si, si, vas-y". Et elle me dit qu'elle a senti qu'elle a eu peur mais aussi une force et elle lui a dit : "Non tu es dégueulasse, je vais parler à mes parents", elle a dit: "Je me suis sentie forte, j'ai pu lui répondre et j'ai pu partir", et elle a pu s'enfuir. [...] C'est intéressant qu'elle ait été exposée au même genre de situation dont elle a pu se sortir. Elle a pu se sortir de cette situation très réelle alors qu'il y avait trop de fantastique dans l'autre. Au début, c'est une jeune fille face à un djinn alors qu'après elle est en face d'un homme. » La psychologue met en contraste un monde fantastique de passivité et le monde «réel» de la seconde situation où la jeune fille agit et se protège. Elle m'explique que le fantastique est le propre du monde de l'enfance, auquel le cheikh n'a fait qu'apposer une lecture religieuse. J'en déduis que Jasmine attribue au religieux la capacité de cacher des douleurs à la conscience des victimes. Aussi, elle exclut la possibilité selon laquelle l'interprétation religieuse du fantastique de l'enfance aurait fourni à la victime un outil pour agir sur le monde réel ; outil qu'elle aurait abandonné après avoir retrouvé sa capacité à agir sur le monde.

De nombreux travaux se sont intéressés à la manière dont les expressions de souffrances sont traduites en termes médicaux par les professionnels de santé (Kleinman et Desjarlais, 1994 ; Fassin, 2010 ; Fassin et Rechtman, 2011). Parce que la maladie mentale est stigmatisante, les professionnels de santé estiment qu'elle est "sous-diagnostiquée ». Ils associent donc à leur diagnostic une démarche d'investigation afin de dépasser les tabous culturels qui empêchent l'accès aux souffrances (Amri, 2012 : 50 ; Hassan et al., $2016: 6$ ). Un travail de traduction permet d'appliquer les mêmes critères d'évaluation à tous les contextes (Shoeb, Weinstein et Mollica, 2007 ; Moussa et al., 2017). Les souffrances sont ensuite objectivées par une pathologie dont les critères sont les réponses passives (ex : pleurer, fumer, regarder de manière excessive les informations, penser aux disparus, dormir). Les pratiques religieuses (prières, lecture du Coran) ont rarement une valeur thérapeutique reconnue (Ghayda Hassan, Kirmayer et Ventevogel, 2015 : 16-17), alors que certaines 
recherches montrent leur potentiel (Hutchinson et Dorsett, 2012: 61). Les dimensions socioculturelles de la souffrance sont évacuées de l'analyse au profit des échelles individuelle et familiale. Justifiée par un souci d'égalité dans l'accès au soin, cette approche peut nourrir un sentiment d'insécurité des réfugiés issus de minorités religieuses (Eghdamian, 2017 : 9-17). Cette approche présuppose donc l'existence d'un espace neutre, coupé du sujet souffrant, à partir duquel l'évaluateur peut produire un objet-souffrance. Cette objectivation a son utilité dans la mesure où elle peut être quantifiée, ce qui permet à la médecine moderne de soigner et réduire les souffrances.

L'objet-souffrance peut par la suite être utilisé par les réfugiés comme un pouvoir. Jasmine me rapporte que la mère exposait l'histoire de sa fille à tout le monde alors que les intervenants avaient insisté auprès d'elle pour que cette expérience ne soit pas «sa porte d'entrée pour tous les autres services ». L'exposition publique d'un sujet tabou, de même que la similarité de certains récits, suscite la suspicion chez les travailleurs humanitaires. Ils craignent que leurs «critères de vulnérabilités » ne soient instrumentalisés pour obtenir des aides indues. L'objet-souffrance constitue donc un paradoxe. Alors qu'il doit produire une certitude pour les humanitaires, son utilisation par les réfugiés pour répondre à leurs besoins en fait l'objet d'une suspicion qui implique un régime de vérification. Le corps est pris comme un matériau empiriquement observable, indicateur extérieur (signifiant) d'un objet-souffrance interne et invisible (signifié). Cette sémiotique des expressions de souffrances procède d'une tradition discursive sceptique de la douleur en sciences sociales dont l'ouvrage The Body in Pain de Elaine Scarry constitue la théorisation (Asad, 2003 : 80-85 ; Crapanzano, 2004 : 80-83). La douleur y est définie comme une expérience de certitude pour soi, mais de doute radical pour l'autre car la douleur physique résiste au langage. La torture exacerbe cette tendance et la confirme puisqu'elle détruit le langage et renvoie le sujet dans un état antérieur, celui des sons et des cris (Scarry, 1985 : 4). Paul Ricoeur s'inscrit dans cette tradition. Il affirme que la souffrance ne fait pas de doute au niveau individuel, exacerbe le soi et crée une coupure avec l'autre. Elle interroge l'autre et lui demande une impossible substitution des places (Ricoeur, 2013). Pourtant, nous allons voir que la douleur est communicable. Le doute ne s'installe que si l'on ressent le besoin urgent de communiquer sa douleur alors que cette communication échoue. Les apports de la théorie de la reconnaissance nous permettent de dépasser l'impasse d'une théorie sceptique de la douleur.

\section{DU SCEPTICISME VERS LA RECONNAISSANCE}

Naïm et Fatima sont originaires de Dera'. Suite au siège de la ville par l'armée syrienne au printemps 2011, ils se sont réfugiés avec leurs enfants dans le quartier de Daouq, en banlieue sud de Beyrouth. J'ai rencontré sa famille grâce à Rana, une amie libanaise qui aide bénévolement des réfugiés ${ }^{3}$. Alors que Naïm décrit le début des événements en Syrie, nous lui demandons si un événement particulier l'a décidé à fuir : "Oui, ils attaquaient les maisons et arrêtaient les gens qui avaient participé aux manifestations et les combattants de l'Armée [syrienne] libre. Ma maison a brûlé, elle est complètement détruite. C'est le régime parce qu'il contrôle l'armée. Il y avait beaucoup d'espions qui prenaient des photos des manifestants. Il y avait des bombardements tous les jours. Imagine un village de 3000 habitants, bombardé tous les jours pendant trois ans avec des barils de $500 \mathrm{~kg}$ ! [...] Moi, ma femme et mes enfants avons quitté le village. Nous étions six sur une moto. Un char était proche, il a vu que nous n'avions pas d'armes, mais il a commencé à nous tirer dessus. Les gens derrière nous sont morts en martyrs. Nous essayions de nous enfuir. On a commencé à entendre des cris venant des mosquées. Il y avait une attaque et tout le monde essayait de fuir avec ses enfants. »

Naïm explique ensuite les difficultés quotidiennes qu'il rencontre, tout particulièrement pour soigner sa fille : «Les Nations-unies ne sont pas utiles du tout. Une juriste m'a dit d'en parler à un avocat. S'ils veulent interviewer quelqu'un pour la télévision, je serai le premier à le faire. Ils ne répondent à aucun appel. Tu sais ce qu'ils font ? Ils connectent le téléphone à l'ordinateur et 
l'ordinateur répond et gaspille ton crédit téléphonique pour rien, jusqu'au moment où tu n'en as plus. Si tu avais dix dollars à dépenser pour les appeler, tu n'aurais pas besoin de les appeler. » Les théories de la reconnaissance et les travaux en anthropologie cités précédemment se démarquent du scepticisme moderne et séculier parce qu'elles postulent une ontologie relationnelle de la souffrance. Ils affirment que séparer la douleur de l'expression qui lui est donnée met en jeu la relation et donc la compréhension. Les travaux en linguistique de Pierce puis leur application en anthropologie par Silverstein nous invitent à distinguer deux types de signification: une signification référentielle qui est indépendante du contexte et une signification indexiale, dépendante du contexte comme les termes « ici » et « maintenant» (Silverstein, 1976). Dans cette perspective, les expressions de souffrance sont des index. Elles définissent l'objet dont on parle (une douleur) et créent un contexte qui demande aux témoins de prendre une position par rapport à cette douleur (la reconnaître et y répondre) (Perdigon, 2010 : 984-86). Elles ne constituent donc pas des signifiants qui renverraient à un objet intérieur, mais une demande de reconnaissance (Das, 1998 : 191-92).

En revanche, les manifestations souffrantes sont facilement invisibilisées ce qui peut donner l'impression que la douleur est incommunicable. Suite à l'entretien, Rana m'interpelle après avoir quitté Naïm. Elle est sympathisante du Parti national socialiste syrien ${ }^{4}$ et le discours de Naïm contre l'armée syrienne l'irrite : «Ça n'a pas de sens ce qu'il raconte, l'armée ne tire pas sur les gens sans armes, elle est là pour les protéger. Elle n'arrête pas tout le monde. Ses propos ne sont pas cohérents, il raconte soudainement être six sur une moto en train de fuir les bombardements avec un char à côté ! » Le positionnement sur la question religieuse de Jasmine, comme le positionnement politique de Rana, jouent un rôle déterminant dans le processus de reconnaissance. Si la reconnaissance de la souffrance permet la relation, sa non-reconnaissance est une violence qui produit des blessures morales. Ainsi, je distingue une perspective sceptique sur la souffrance, pratiquée par les travailleurs humanitaires, de celle de la reconnaissance que j'adopte ici. Dans la partie suivante, je m'intéresserai à la notion d'empathie en phénoménologie afin d'enrichir encore la discussion critique de cette conception sceptique.

\section{VERS UNE EMPATHIE PHÉNOMÉNOLOGIQUE}

Il n'est pas possible de présenter ici une histoire européenne des théories d'accès aux souffrances d'autrui. Rappelons simplement qu'avant les travaux de Boltanski (2007) et de Fassin (2010) sur le rôle du sentimentalisme moral des Lumières dans l'action « humanitaire », l'anthropologie postmoderne et post-coloniale soulignait déjà le rôle central de la sympathie dans la construction morale de l'idée moderne d' «humanité ». Les Lumières voient le remplacement d'un universalisme chrétien de compassion par celui de la sympathie naturelle et séculière. Dans cette nouvelle conception, le sujet reconnaît sa souffrance dans le spectacle de la souffrance d'autrui. La sympathie lui permet de généraliser sa condition à l'humanité et de développer un sens moral universel. Seules les coutumes particulières considérées comme "barbares » peuvent empêcher l'expression de sa sympathie et le progrès moral qui lui est associé (Chakrabarty, 2000 : 52-59). Or, un des objectifs du progrès moderne est de réduire la douleur de l'humanité. Par conséquent l'objectivation et la quantification des douleurs rapportées à une logique utilitariste permet de justifier une certaine violence (Asad, $2003: 67-84 ; 107-17$ ).

Il possible d'inscrire la conception moderne de la sympathie dans une des deux branches de la théorie de l'esprit qui regroupe l'ensemble des approches traitant de la capacité à concevoir ses états mentaux et ceux des autres. La « théorie de la théorie » postule d'une impossibilité pour l'individu d'accéder complètement à propos esprit pour affirmer que nous utilisons une théorie (innée pour certains auteurs, acquise pour d'autres) afin de déduire la signification du comportement d'autrui. Selon la " théorie simulationiste », c'est au contraire l'accès à nos états mentaux qui nous permet d'imaginer ceux des autres par imitation (Zahavi, 2014 : 99-106). Cette dernière se retrouve dans un 
ensemble de conceptions allant d'Aristote jusqu'à la sympathie moderne puis chez Wittgenstein et dans l'approche de Das (Das, 2007 : 39-57). Or, c'est bien l'imagination qui a le potentiel de produire des blessures morales. Elle permet de se représenter soi-même en train de mentir sur ses douleurs et donc d'attribuer aux autres cette même capacité (Kelly, 2012).

L'anthropologie, dans la lignée de Geertz, a longtemps refusé de «prendre la place » de ses sujets d'étude afin d'éviter de projeter ses états sur l'autre. L'empathie husserlienne telle qu'appliquée en anthropologie permet de limiter ce risque. Elle se définit comme : « un processus intersubjectif et imaginatif qui comprend aussi bien l'expérience de comprendre l'autre que d'être compris par lui » (Throop, 2010: 772). L'échange de position (Platzwechsel) permet d'établir un jumelage (Paarung). Mon expérience me permet de comprendre l'autre mais elle est également modifiée par la rencontre ce qui me permet d'acquérir de nouvelles expériences. Elle requiert une implication émotionnelle et personnelle du chercheur que chacun peut légitiment refuser. Faire l'expérience de l'autre, c'est se laisser affecter pour permettre un échange qui n'était pas prévu ou intentionnel (Favret-Saada, 1990).

L'adoption d'une théorie de la reconnaissance complétée par une empathie phénoménologique offre donc des perspectives théoriques intéressantes pour l'étude des dimensions socioculturelles de la souffrance. Elles permettent de conceptualiser le rôle de l'empathie dans les processus d'engagement comme nous allons le voir dans cette dernière partie.

\section{S'ENGAGER PAR EMPATHIE}

Pour cela, revenons au récit de Naïm et Fatima. Cette dernière décrit ses difficultés quotidiennes : «La dernière fois, je suis allée à la pharmacie. Il y avait quelqu'un qui voulait prendre ma place dans la file d'attente. Il m'a attrapé par l'épaule alors que je portais un manteau et mon voile (hijab) et il m'a dit: "Vous les Syriens vous ne comprenez pas, on est obligé d'être impoli avec vous". Je l'ai regardé et il m'a dit de sortir. Je lui ai dit: "Ce ne sont pas vos affaires, vous n'êtes pas le propriétaire de cette pharmacie et je paye ces médicaments avec mon propre argent". Ils ne nous respectent pas du tout. Il y a beaucoup d'histoire dans le quartier. Ma voisine, je ne veux pas dire son nom, elle met intentionnellement ses ordures devant notre porte chaque jour. Je dois les enlever tous les jours. Elle est libanaise et n'aime pas les Syriens. » Fatima a sollicité le sens éthique de son interlocuteur pour qu'il reconnaisse qu'elle aussi a des préoccupations éthiques (Honneth, 2005 ; Ferrarese, 2009: 106-7). Une demande de reconnaissance participe donc à la formation de la dignité, comprise comme une image positive de soi. Lorsque les souffrances sont invisibilisées, elles rompent les relations entre les individus mais peuvent également susciter l'indignation. Les accusations, comme celles de Naïm, ainsi que les insultes, constituent des processus d'activation de la souffrance. Ils permettent aux réfugiés de faire face aux difficultés de l'exil en luttant pour leur reconnaissance.

Certains réfugiés vont au-delà et s'engagent pour répondre aux souffrances de leurs pairs. Abou Dalil est un électricien de 50 ans originaire de la côte syrienne ${ }^{5}$. Pendant les six premiers mois de la révolution, il a participé aux manifestations pacifiques et distribué de l'aide : «Nous distribuions des médicaments et de la nourriture dans les villages bombardés. Le gouvernement ne nous disait rien. Ils disaient qu'il y avait des miliciens à l'intérieur des villages et qu'ils nettoyaient et qu'ils envoyaient de l'aide. Et puis ils ont commencé à arrêter les gens. Mon cousin a été arrêté, son corps m'a été rendu trois jours après qu'ils aient brisé son cou. » Il a quitté la Syrie lorsqu'il a appris que les services de sécurité le recherchaient. Il s'estime trop vieux pour combattre, il est devenu infirmier pour un centre médical au Liban qui a été mis en place grâce au soutien financier de Syriens expatriés et de riches nationaux du Golfe. Lorsque je lui demande s'il préfère son travail d'électricien ou d'infirmier, il me répond : « C'est incomparable. Ce que je fais maintenant me plaît vraiment. Tu n'as pas à penser à la révolution et les directions dans lesquelles elle va. Tu dois penser à ce que tu donnes pour la révolution. » 
Abou Dalil travaille sans interruption depuis un an et demi. Cela lui permet de «ne pas penser» à sa famille qu'il a été obligé de laisser en Syrie. Il accuse le régime d'avoir manipulé le mouvement pour en faire une opposition armée qu'il a pu légitimement réprimer : "Tu avais peur d'être arrêté ou torturé ? Personne n'a peur de la mort mais la peur d'être arrêté et torturé est la pire chose qui puisse t'arriver. Mais comment manifester alors ? Les gens en avaient assez de la situation, ils étaient étouffés (makhnouqin). Au début, les gens arrêtés sortaient mais après ils sortaient avec un handicap ou alors ils étaient fous et cela a fait changer les gens. Et puis le sang, quand ton frère est touché par une balle, meurt devant toi et que tu as vu le tueur, tu n'as plus peur de rien. »

La confrontation avec la violence du régime est présentée par Abou Dalil comme une rupture pour tous les Syriens, mais la perte de son cousin puis l'exil à distance de sa famille constituent des ruptures personnelles. Je les analyse comme les événements d'un processus de changement de perspective éthique. Dans ses travaux sur l'éthique, Webb Keane avance l'hypothèse selon laquelle des changements dans la conscience éthique peuvent être déclenchés par le conflit social (2015: 186-96). Le conflit, s'il est l'objet d'une pratique et pas seulement d'une contemplation, peut amener l'individu à prendre de la distance avec sa perspective (à la $1^{\text {re }}$ personne) pour se voir à travers le regard des autres ( $3^{\mathrm{e}}$ personne). Le partage puis la vérification des émotions individuelles au sein d'un groupe permettent d'évacuer le doute. La reconnaissance mutuelle produit une éthique partagée. Par exemple, la colère devient une émotion légitime à exprimer en public alors qu'elle était auparavant contenue, ce qui explique qu'elle ait remplacé la peur de la répression selon Abou Dalil. Je considère donc ce processus comme une discipline sociale de la pratique de l'empathie. Elle a des conséquences intérieures pour le sujet qui ouvre des possibilités de mobilisation politique. Ainsi, il me semble que la notion d'empathie vient nourrir tant l'élaboration d'une épistémologie de la souffrance en sciences sociales que notre compréhension des processus d'engagement à la suite d'événements de rupture. 

2016) : Le rôle du religieux et du politique dans la formation d'éthiques de la souffrance ». Son matériau est constitué d'observations menées dans les différentes régions du Liban (Beyrouth, Tripoli, Beka'a, Chouf, Akkar) ainsi que d'une centaine de témoignages rassemblés lors d'une présence continue au Liban pendant quatre années. Les 92 heures d'entretiens ont été enregistrées en arabe dialectal avec l'aide d'intermédiaires puis retranscrites et traduites vers le français. Deux années ont été financées par une bourse d'Aide à la mobilité internationale de l'Institut français du proche-orient à Beyrouth.

2 . Entretien réalisé en français le 03/12/2015 à Mansourieh, Liban.

3 . Entretien réalisé en arabe levantin le 16/03/2014 à Daouq, Liban.

4 . Le PNSS est un parti qui défend l'unité arabe, impliqué dans la guerre civile libanaise du côté des Palestiniens et aujourd'hui du côté du régime ba'thiste en Syrie.

5 . Entretien réalisé le 14/04/2014 à Tripoli en arabe levantin, Liban.

\section{Bibliographie}

Amri, S. 2012, « Mental Health Help-Seeking Behaviors of Muslim Immigrants in the United States: Overcoming Social Stigma and Cultural Mistrust » dans Journal of Muslim Mental Health, vol. 7, no $1: 43-63$.

Asad, T. 2003, Formations of the Secular: Christianity, Islam, Modernity, Stanford, Stanford University Press.

Boltanski, L. 2007, La souffrance à distance, Paris, Folio.

Chakrabarty, D. 2000, «Witness to Suffering: Domestic Cruelty and the Birth of the Modern Subject in Bengal » dans Mitchell, T. (éd) Questions of Modernity, Minneapolis, University of Minnesota Press, pp. 49-86.

Crapanzano, V. 2004, Imaginative Horizons: An Essay in Literary-Philosophical Anthropology, Chicago, University of Chicago Press.

Das, V. 2007, Life And Words: Violence And the Descent Into the Ordinary, Berkeley, University of California Press.

---. 2016, « The Boundaries of the "We" Cruelty, Responsibility and Forms of Life » dans Critical Horizons, vol. 17, $\mathrm{n}^{\circ} 2$ : 168-85.

---. 1998, « Wittgenstein and Anthropology » dans Annual Review of Anthropology, vol. 27, $\mathrm{n}^{\mathrm{o}} 1: 171-95$.

Eghdamian, K. 2017, « Religious Identity and Experiences of Displacement: An Examination into the Discursive Representations of Syrian Refugees and Their Effects on Religious Minorities Living in Jordan » dans Journal of Refugee Studies, vol. 30, $\mathrm{n}^{\mathrm{O}}$ $3: 447-67$.

Fassin, D. 2010, La Raison Humanitaire: Une Histoire Morale du Temps Présent, Paris, Gallimard.

Fassin, D. et Rechtman, R. 2011, L'empire du traumatisme: Enquête sur la condition de victime, Paris, Flammarion.

Favret-Saada, J. 1990, « Être affecté » dans Grandhiva, n $8:$ 3-10.

Ferrarese, E. 2009, « Qu'est-ce qu'une lutte pour la reconnaissance? : Réflexions sur l'antagonisme dans les théories contemporaines de la reconnaissance » dans Politique et Société, vol. 28, n 3 : 101-16.

Hassan, G., et al. 2016, « Mental Health and Psychosocial Wellbeing of Syrians Affected by Armed Conflict » dans Epidemiology and Psychiatric Sciences, vol. 25, n ${ }^{\circ} 2: 129-41$. Hassan, G, et al. 2015, Culture, Context and the Mental Health and Psychosocial Wellbeing of Syrians: A Review for Mental Health and Psychosocial Support Staff Working with Syrians Affected by Armed Conflict, Genève, United Nations High Commissioner for Refugees.

Honneth, A. 2005, « Invisibilité: sur l'épistémologie de la «reconnaissance» » dans Réseaux, vol. $\mathrm{n}^{\circ} 129-130, \mathrm{n}^{\circ} 1: 39-57$.

Hutchinson, M. et Dorsett, P. 2012, « What Does the Literature Say about Resilience in Refugee People? Implications for Practice » in Journal of Social Inclusion, vol. 3, $\mathrm{n}^{\circ} 2$ : 55-78. 
Keane, W. 2015, Ethical Life: Its Natural and Social Histories, Princeton, Princeton University Press.

Kelly, T. 2012, « Sympathy and Suspicion: Torture, Asylum, and Humanity » dans Journal of the Royal Anthropological Institute, vol. 18, $\mathrm{n}^{\circ} 4$ : 753-68.

Kleinman, A. et Desjarlais, R. 1994, « Ni patients ni victimes Pour une ethnographie de la violence politique » dans Actes de la recherche en sciences sociales, vol. 104, $\mathrm{n}^{\circ} 1$ : 56-63. Moussa, M. et al. 2017, « Psychometric Properties of an Arabic Version of the Depression Anxiety Stress Scales (DASS) » dans Research on Social Work Practice, vol. 27, n 3 : 375-86.

Perdigon, S. 2010, « L'ethnographie à l'heure des martyrs Histoire, violence, souffrance dans la pratique anthropologique contemporaine » dans Annales. Histoire, Sciences Sociales, vol. 65e année, $\mathrm{n}^{\circ} 4$ : 971-96.

Ricoeur, P. 2013, « La souffrance n'est pas la douleur » dans Souffrance et douleur. Autour de Paul Ricoeur, édité par Claire Marin \& Nathalie Zaccai-Reyners (éds), Paris, Presses Universitaires de France, pp. 13-33.

Scarry, E. 1985, The Body in Pain: The Making and Unmaking of the World, Oxford, Oxford University Press.

Shoeb, M. et al. 2007, « The Harvard Trauma Questionnaire: Adapting a Cross-Cultural Instrument for Measuring Torture, Trauma and Posttraumatic Stress Disorder in Iraqi Refugees » dans International Journal of Social Psychiatry, vol. 53, $\mathrm{n}^{\circ} 5$ : 447-63. Silverstein, M. 1976, « Shifters, linguistic categories, and cultural description » dans Meaning in Anthropology, édité par Keith H. \& Selby, H. (éds), Albuquerque, University of New Mexico Press, pp. 11-55.

Throop, C.J. 2010, « Latitudes of Loss: On the Vicissitudes of Empathy » dans American Ethnologist, vol. 37, $\mathrm{n}^{\circ} 4:$ :771-82.

Zahavi, D. 2014, Self and Other: Exploring Subjectivity, Empathy, and Shame, Oxford, Oxford University Press 